\title{
Telegraphy Equation From Weber's Electrodynamics
}

\author{
A. K. T. Assis and J. A. Hernandes
}

\begin{abstract}
We derive the telegraphy equation according to Weber's electrodynamics for signal propagating along a very long bidimensional wire in the shape of a rectangular strip of zero thickness. We also derive this equation for a twin lead composed by two of these parallel very long bidimensional wires facing each other. We compare this result with classical electromagnetism.
\end{abstract}

Index Terms-Circuit theory, propagation of electromagnetic signals, telegraphy equation, twin leads, Weber's electrodynamics.

\section{INTRODUCTION}

$\mathbf{T}$ HE FIRST TO DERIVE the telegraphy equation taking into account the capacitance, self-inductance and resistance of the wire were Weber and Kirchhoff in the year 1857 [1, vol. 1, pp. 144-146, 296-297], [2], [3], [4, , pp. 230-232], and [5, Sec. 3.1]. They published independent works but both of them were based on Weber's action at a distance theory. For a wire of negligible resistance, they showed that the signal would propagate at light velocity. Kirchhoff's original papers are [6] and [7], with English translations in, respectively, [8] and [9]. Weber's simultaneous and more thorough work was delayed in publication and appeared only in 1864 [10]. It was only in 1876 that Heaviside succeeded in deriving the same equation based on Maxwell's equations [4, pp. 228-229].

Weber and Kirchhoff considered the propagation of signals in conducting wires of circular cross sections. Our goal here is to extend their works for signals propagating through a long rectangular strip and along a twin lead composed of two of these strips. We will also compare this result based on Weber's electrodynamics with classical electromagnetism. Modern theoretical and experimental discussion of Weber's electrodynamics can be found in [5], [11]-[15], [16, ch. 6], and [17]-[26].

Suppose a charge element $d q_{2}$ located at $\vec{r}_{2}$, moving with velocity $\vec{v}_{2}$ and acceleration $\vec{a}_{2}$ relative to an inertial frame of reference $S$, and a point charge $q_{1}$ located at $\vec{r}_{1}$, moving with velocity $\vec{v}_{1}$ and acceleration $\vec{a}_{1}$ relative to $S$. According to Weber's electrodynamics the force exerted by 2 on 1 is given by [5, ch. $3]$ and $[24$, ch. 8]

$$
\begin{array}{r}
d \vec{F}=\frac{q_{1} d q_{2}}{4 \pi \varepsilon_{o}} \frac{\hat{r}_{12}}{r_{12}^{2}}\left[1+\frac{1}{c^{2}}\left(\vec{v}_{12} \cdot \vec{v}_{12}-\frac{3}{2}\left(\hat{r}_{12} \cdot \vec{v}_{12}\right)^{2}\right.\right. \\
\left.\left.+\vec{r}_{12} \cdot \vec{a}_{12}\right)\right]
\end{array}
$$

Manuscript received October 10, 2003; revised September 28, 2004. The work of J. A. Hernandes was supported by CNPq, Brazil. The work of A. K. T. Assis supported by the Alexander von Humboldt Foundation, Germany. This paper was recommended by Associate Editor M. P. Kennedy.

The authors are with the Instituto de Fílsica "Gleb Wataghin," Universidade Estadual de Campinas-Unicamp, 13083-970 São Paulo, Brazil (e-mail: assis@ifi.unicamp.br; julioher@ifi.unicamp.br).

Digital Object Identifier 10.1109/TCSII.2005.848958

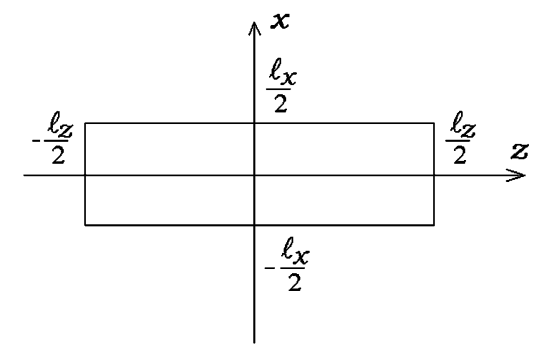

Fig. 1. Geometry of the problem.

where $\varepsilon_{o}=8.85 \times 10^{-12} \mathrm{C}^{2} \mathrm{~N}^{-1} \mathrm{~m}^{-2}$ is the permittivity of vacuum, $c=3 \times 10^{8} \mathrm{~ms}^{-1}, \vec{r}_{12}=\vec{r}_{1}-\vec{r}_{2}, \vec{v}_{12}=\vec{v}_{1}-\vec{v}_{2}$, $\vec{a}_{12}=\vec{a}_{1}-\vec{a}_{2}, r_{12}=\left|\vec{r}_{12}\right|$, and $\hat{r}_{12}=\vec{r}_{12} / r_{12}$ is the unit vector pointing from 2 to 1 . This is the basic expression we will employ here, integrating it over the wire.

\section{RECTANGULAR STRIP}

The first case to be considered is that of a long rectangular strip of length $\ell_{z}$ and width $\ell_{x}$ in the $x z$ plane centered on the origin of the coordinate system, with the longer side $\ell_{z}$ along the $z$ axis, Fig. 1. We suppose air or vacuum outside this bidimensional conductor. As we have a bidimensional problem an element of source charge is given by $d q_{2}=\sigma_{f}\left(x_{2}, 0, z_{2}, t\right) d x_{2} d z_{2}$, where $\sigma_{f}\left(x_{2}, 0, z_{2}, t\right)$ is the surface density of free charges at the position $\left(x_{2}, 0, z_{2}\right)$ of the strip in time $t$, and $d x_{2} d z_{2}$ is an element of area. As regards the velocity and acceleration of the source charges we will assume a longitudinal current and propagation, namely $\vec{v}_{2}\left(x_{2}, 0, z_{2}, t\right)=v_{2}\left(z_{2}, t\right) \hat{z}$ and $\vec{a}_{2}\left(x_{2}, 0, z_{2}, t\right)=a_{2}\left(z_{2}, t\right) \hat{z}$. The test charge will be located at $\left(x_{1}, y_{1}, z_{1}, t\right)$, in such a way that its distance to the source charge $d q_{2}$ is given by $r_{12}=\sqrt{\left(x_{1}-x_{2}\right)^{2}+y_{1}^{2}+\left(z_{1}-z_{2}\right)^{2}}$.

The first component of Weber's force acting on charge $q_{1}$ is the Coulomb force which can be obtained as the gradient of a potential $\phi,-q_{1} \nabla_{1} \phi\left(x_{1}, y_{1}, z_{1}, t\right)$, where

$$
\begin{aligned}
& \phi\left(x_{1}, y_{1}, z_{1}, t\right)=\frac{1}{4 \pi \varepsilon_{o}} \\
& \int_{-\ell_{x} / 2}^{\ell_{x} / 2} \int_{-\ell_{z} / 2}^{\ell_{z} / 2} \frac{\sigma_{f}\left(x_{2}, 0, z_{2}, t\right) d x_{2} d z_{2}}{\sqrt{\left(x_{1}-x_{2}\right)^{2}+y_{1}^{2}+\left(z_{1}-z_{2}\right)^{2}}} .
\end{aligned}
$$

Here, $\phi\left(x_{1}, y_{1}, z_{1}, t\right)$ is the potential at the location of $q_{1}$ due to all source charges $d q_{2}$.

In order to solve this equation, we utilize the approximation

$$
\ell_{z} \gg \ell_{x} \gg r_{1}
$$

where $r_{1}=\sqrt{x_{1}^{2}+y_{1}^{2}+z_{1}^{2}}$ is the distance of the test charge to the center of the strip. 
We now employ Kirchhoff's main idea in order to solve this integral. For any given $r_{1}$ and $r_{2}$, the maximum value of $1 / r_{12}=1 / \sqrt{\left(x_{1}-x_{2}\right)^{2}+y_{1}^{2}+\left(z_{1}-z_{2}\right)^{2}}$ is for $x_{1}=x_{2}$ and $z_{1}=z_{2}$, when $1 / r_{12}=1 /\left|y_{1}\right|$. For $z_{2}$ far from $z_{1}$, the value of $r_{12}$ will be close to $\left|z_{2}\right|$ due to the approximation (3). In this case, $\left|z_{2}\right|$ will be of the order of $\ell_{z}$, namely $1 / r_{12} \approx 1 /\left|z_{2}\right| \approx 1 / \ell_{z}$. By approximation (3) we have $1 /\left|y_{1}\right| \gg 1 / \ell_{z}$. Kirchhoff's idea is then to remove $\sigma_{f}\left(x_{2}, 0, z_{2}, t\right)$ from the integrand by taking its value at the maximum value of $1 / r_{12}$, that is, when $z_{2}=z_{1}$ and $x_{2}=x_{1}$. We are then led to the approximate result (see Appendix)

$$
\begin{aligned}
\phi\left(x_{1}, y_{1}, z_{1}, t\right) \approx & \frac{\sigma_{f}\left(x_{1}, 0, z_{1}, t\right)}{4 \pi \varepsilon_{o}} \int_{-\ell_{x} / 2}^{\ell_{x} / 2} \int_{-\ell_{z} / 2}^{\ell_{z} / 2} \\
& \times \frac{d x_{2} d z_{2}}{\sqrt{\left(x_{1}-x_{2}\right)^{2}+y_{1}^{2}+\left(z_{1}-z_{2}\right)^{2}}} \\
\approx & \frac{\sigma_{f}\left(x_{1}, 0, z_{1}, t\right) \ell_{x}}{2 \pi \varepsilon_{o}} \ln \frac{\ell_{z}}{\ell_{x}} .
\end{aligned}
$$

To solve this last integral, we integrated first in $z_{2}$ and utilized once more approximation (3).

The longitudinal Coulomb force is then given by (with $\vec{F}=$ $\left.-q_{1} \nabla_{1} \phi\right)$

$$
F_{z}=-\frac{q_{1} \ell_{x}}{2 \pi \varepsilon_{o}} \frac{\partial \sigma_{f}}{\partial z_{1}} \ln \frac{\ell_{z}}{\ell_{x}} .
$$

The terms proportional to $v_{2}^{2} / c^{2}$ and to $\left(\hat{r}_{12} \cdot \vec{v}_{2}\right)^{2} / c^{2}$ are usually small compared to the Coulomb term and will be neglected here. The reason for this is that $\left|v_{2}\right|$ is of the order of the drifting velocity $v_{d}$, which is known to be much smaller than the light velocity $c$. The terms depending on $\vec{v}_{1} \cdot \vec{v}_{2}$ and $\left(\hat{r}_{12} \cdot \vec{v}_{1}\right)\left(\hat{r}_{12} \cdot \vec{v}_{2}\right)$ give rise to the magnetic force $q_{1} \vec{v}_{1} \times \vec{B}[5$, Sec 6.6, 7.4] and [27]-[30]. As the current is in the longitudinal direction $\hat{z}$, the magnetic field on the strip will be in the $y$ direction. The test charge considered here will be a conduction electron on the strip moving in the $\hat{z}$ direction, so that $q_{1} \vec{v}_{1} \times \vec{B}$ will be in the $x$ direction. As we are interested only in the longitudinal propagation of the signal along the $z$ direction, we will not consider these terms either.

We then need to take into account the acceleration term of Weber's force acting on the test charge $q_{1}$ due to the accelerated conduction electrons, which can be written as

$$
\begin{aligned}
\vec{F}= & -\frac{q_{1}}{4 \pi \varepsilon_{o} c^{2}} \int_{-\ell_{x} / 2}^{\ell_{x} / 2} \int_{-\ell_{z} / 2}^{\ell_{z} / 2} \\
& \times \frac{\left(x_{1}-x_{2}\right) \hat{x}+y_{1} \hat{y}+\left(z_{1}-z_{2}\right) \hat{z}}{\left[\left(x_{1}-x_{2}\right)^{2}+y_{1}^{2}+\left(z_{1}-z_{2}\right)^{2}\right]^{3 / 2}} \\
& \times\left(z_{1}-z_{2}\right) a_{2}\left(z_{2}, t\right) \sigma_{c} d x_{2} d z_{2} .
\end{aligned}
$$

Here, $\sigma_{c}$ is the surface density of conduction electrons (typically one electron per atom in copper conductors). It has a constant value which does not depend on position. This means that it can be removed from the integrand.

By Kirchhoff's approximation method, we can remove $a_{2}\left(z_{2}, t\right)$ from the integrand taking its value at $x_{2}=x_{1}$ and $z_{2}=z_{1}$ (that is, at the maximum value of $1 / r_{12}$ ). Considering only the $z$ component of the force we obtain the approximate result (utilizing also that $c^{2}=1 / \mu_{o} \varepsilon_{o}$ )

$$
\begin{aligned}
F_{z} \approx & -\frac{\mu_{o}}{4 \pi} q_{1} \sigma_{c} a_{2}\left(z_{1}, t\right) \int_{-\ell_{x} / 2}^{\ell_{x} / 2} \int_{-\ell_{z} / 2}^{\ell_{z} / 2} \\
& \times \frac{\left(z_{1}-z_{2}\right)^{2} d x_{2} d z_{2}}{\left[\left(x_{1}-x_{2}\right)^{2}+y_{1}^{2}+\left(z_{1}-z_{2}\right)^{2}\right]^{3 / 2}} \\
\approx & -\frac{\mu_{o}}{2 \pi} q_{1} \ell_{x} \sigma_{c} a_{2} \ln \frac{\ell_{z}}{\ell_{x}} .
\end{aligned}
$$

We now suppose the test charge to be a conduction electron: $q_{1}=-e=-1.6 \times 10^{-19} \mathrm{C}, \vec{v}_{1}=v_{2}\left(z_{1}, t\right) \hat{z}$, and $\vec{a}_{1}=a_{2}\left(z_{1}, t\right) \hat{z}$. In this case we must also include the frictional force due to its collisions with the lattice. The average value of this force can be represented by $-b \vec{v}_{1}$, where the coefficient of friction $b$ is given by $b=q_{1} \sigma_{c} \ell_{x} R / \ell_{z}$, with $R$ being the resistance of the strip, [31]. This is the necessary value of $b$ in order to obtain Ohm's law for constant current in a bidimensional conductor.

We can now write down the $z$ component of the equation of motion for a conduction electron applying Newton's second law of motion in the longitudinal direction $z$, namely: $F_{z}=m a$. Considering the frictional force plus (5) and (7) in Newton's second law of motion, dropping the subscript 1 and writing $q_{1}=$ $-e$ yields

$$
\frac{e \ell_{x}}{2 \pi \varepsilon_{o}} \frac{\partial \sigma_{f}}{\partial z} \ln \frac{\ell_{z}}{\ell_{x}}+\frac{e \ell_{x} \mu_{o}}{2 \pi} \sigma_{c} a \ln \frac{\ell_{z}}{\ell_{x}}+e \sigma_{c} R \frac{\ell_{x}}{\ell_{z}} v=m a .
$$

Usually $\left|e \ell_{x} \mu_{o} \sigma_{c} \ln \left(\ell_{z} / \ell_{x}\right) /(2 \pi)\right| \gg m$, [31], so that we can neglect the term $m a$ in this equation compared with the term $e \ell_{x} \mu_{o} \sigma_{c} \ln \left(\ell_{z} / \ell_{x}\right) a /(2 \pi)$. For instance, for a $1-\mathrm{m}$ wire with 1-mm width, we have, with $e=1.6 \times 10^{-19} \mathrm{C}$ and $\sigma_{c} \approx-1$ $\mathrm{Cm}^{-2}$ for copper: $\left|e \ell_{x} \mu_{o} \sigma_{c} \ln \left(\ell_{z} / \ell_{x}\right) /(2 \pi)\right| \approx 2 \times 10^{-28} \mathrm{~kg}$, which is much greater than the electron mass $m=9 \times 10^{-31}$ $\mathrm{kg}$. Neglecting for this reason the term ma we can then equate the left-hand side of (8) to zero.

We can relate the drifting velocity of the source charges $v_{2}$ with the surface current density $K$ utilizing $K\left(z_{2}, t\right)=$ $\sigma_{c} v_{2}\left(z_{2}, t\right)$. As the surface density of conduction electrons has a constant value we have $\partial K / \partial t=\sigma_{c} a_{2}$. Utilizing this in (8) without the right-hand side, multiplying it by $\left(2 \pi \varepsilon_{o}\right) /\left(e \ell_{x} \ln \left(\ell_{z} / \ell_{x}\right)\right)$ and utilizing $c^{2}=1 / \mu_{o} \varepsilon_{o}$ yields

$$
\frac{\partial \sigma_{f}}{\partial z}+\frac{1}{c^{2}} \frac{\partial K}{\partial t}+\frac{2 \pi \varepsilon_{o} R K}{\ell_{z} \ln \left(\ell_{z} / \ell_{x}\right)}=0 .
$$

There are two unknowns in this equation, $\sigma_{f}$ and $K$. In order to relate them we utilize the equation for the conservation of charges, $\nabla \cdot \vec{J}=-\partial \rho_{f} / \partial t$. For the case considered here of a current flowing in the $z$ direction over the surface of a strip of length $\ell_{z}$ and width $\ell_{x}$ this is equivalent to

$$
\frac{\partial K}{\partial z}=-\frac{\partial \sigma_{f}}{\partial t}
$$


Applying $\partial / \partial t$ in (9) and utilizing (10) yields

$$
\frac{\partial^{2} K}{\partial z^{2}}-\frac{1}{c^{2}} \frac{\partial^{2} K}{\partial t^{2}}=\frac{2 \pi \varepsilon_{o} R}{\ell_{z} \ln \left(\ell_{z} / \ell_{x}\right)} \frac{\partial K}{\partial t} .
$$

This is the equation of telegraphy, which will also be satisfied by $\sigma_{f}$, by $\phi$ and by the total current $I=\ell_{x} K$.

If the resistance of the strip is negligible, Weber's electrodynamics plus Newton's second law of motion predicts a current flow obeying the wave equation. That is, with a signal propagating at light velocity.

\section{TWIN LEAD}

We now perform the same calculations as above but considering a twin lead composed of two of these strips facing one another and separated by a distance $d$ such that $\ell_{z} \gg \ell_{x} \gg d$, with currents flowing longitudinally along the $z$ axis. The parallel planes of the strips are located at $y=d / 2$ and at $y=-d / 2$.

Beyond the conditions above, we need only two further relations connecting the free surface charge densities and surface current densities in both strips. The most reasonable conditions are that (see [32, pp. 125-130]

$$
\sigma_{f,-d / 2}(z, t)=-\sigma_{f, d / 2}(z, t)=\sigma_{f}(z, t)
$$

and

$$
K_{-d / 2}(z, t)=-K_{d / 2}(z, t)=K(z, t) .
$$

The approximation utilized here is

$$
\ell_{z} \gg \ell_{x} \gg x_{1}, y_{1}, z_{1}, d .
$$

With these conditions, integrating for both strips as in the previous Section and utilizing Kirchhoff's approximation method yields

$$
\begin{aligned}
\phi(y \geq d / 2) & =\frac{\sigma_{f} d}{2 \varepsilon_{o}}\left(1-\frac{4 y}{\pi \ell_{x}}\right) \\
\phi(-d / 2 \leq y \leq d / 2) & =\frac{\sigma_{f} y}{\varepsilon_{o}}\left(1-\frac{2 d}{\pi \ell_{x}}\right) \\
\phi(y \leq-d / 2) & =-\frac{\sigma_{f} d}{2 \varepsilon_{o}}\left(1+\frac{4 y}{\pi \ell_{x}}\right) .
\end{aligned}
$$

The longitudinal Coulomb force given by $F_{z}=-q_{1} \partial \phi / \partial z$ yields

$$
\begin{aligned}
F(y \geq d / 2) & =-\frac{q_{1} d}{2 \varepsilon_{o}}\left(1-\frac{4 y}{\pi \ell_{x}}\right) \frac{\partial \sigma_{f}}{\partial z} \\
F(-d / 2 \leq y \leq d / 2) & =-\frac{q_{1} y}{\varepsilon_{o}}\left(1-\frac{2 d}{\pi \ell_{x}}\right) \frac{\partial \sigma_{f}}{\partial z} \\
F(y \leq-d / 2) & =\frac{q_{1} d}{2 \varepsilon_{o}}\left(1+\frac{4 y}{\pi \ell_{x}}\right) \frac{\partial \sigma_{f}}{\partial z} .
\end{aligned}
$$

The longitudinal component of Weber's force depending on the acceleration yields

$$
\begin{aligned}
F(y \leq d / 2) & =-\frac{q_{1} \mu_{o} d}{2}\left(1-\frac{4 y}{\pi \ell_{x}}\right) \frac{\partial K}{\partial t} \\
F(-d / 2 \leq y \leq d / 2) & =-q_{1} \mu_{o} y\left(1-\frac{2 d}{\pi \ell_{x}}\right) \frac{\partial K}{\partial t} \\
F(y \leq-d / 2) & =\frac{q_{1} \mu_{o} d}{2}\left(1+\frac{4 y}{\pi \ell_{x}}\right) \frac{\partial K}{\partial t} .
\end{aligned}
$$

Following the procedure of the previous Section, substituting $b$ by the expression $q_{1} \sigma_{c} R_{1} \ell_{x} / \ell_{z}$ (where $R_{1}$ is the resistance of one strip, [31]) and $q_{1}$ by $-e$ yields

$$
\frac{e d}{2 \varepsilon_{o}} \frac{\partial \sigma_{f}}{\partial z}+\frac{\mu_{o} e d}{2} \frac{\partial K}{\partial t}+\frac{e R_{1} \ell_{x}}{\ell_{z}} K=m a .
$$

Utilizing once more (10) and neglecting the right-hand side of (24) yields

$$
\frac{\partial^{2} K}{\partial z^{2}}-\frac{1}{c^{2}} \frac{\partial^{2} K}{\partial t^{2}}=\frac{\varepsilon_{o} R \ell_{x}}{\ell_{z} d} \frac{\partial K}{\partial t}
$$

where $R=2 R_{1}$ is the resistance of the system of two equal strips.

The same equation is obtained for $\sigma_{f}$, for $\phi$ and for the total current $I=\ell_{x} K$.

\section{Classical Electromagnetism}

Equations (11) and (25) can be put in the form

$$
\frac{\partial^{2} K}{\partial z^{2}}-\frac{L C}{\ell_{z}^{2}} \frac{\partial^{2} K}{\partial t^{2}}=\frac{R C}{\ell_{z}^{2}} \frac{\partial K}{\partial t}
$$

where $R, L$, and $C$ are the resistance, self-inductance, and capacitance of the system. This can be seen observing that the self-inductance and capacitance of a single strip of length $\ell_{z}$ and width $\ell_{x}$, with $\ell_{z} \gg \ell_{x}$, are given by, respectively, $L=$ $\left(\mu_{o} / 2 \pi\right) \ell_{z} \ln \left(\ell_{z} / \ell_{x}\right)$ and $C=2 \pi \varepsilon_{o} \ell_{z} / \ln \left(\ell_{z} / \ell_{x}\right)$. In the case of the twin-lead considered above, the values of these quantities are $L=\mu_{o} \ell_{z} d / \ell_{x}$ and $C=\varepsilon_{o} \ell_{x} \ell_{z} / d$.

Equation (26) is the equation of telegraphy obtained in classical electromagnetism, [4, p. 229] and [33, p. 318]. We then conclude that as regards the telegraphy equation for long straight strips or for twin leads composed of these strips we obtain the same result with classical electromagnetism and Weber's electrodynamics.

\section{APPENDIX}

Herein, we calculate the integral that appears in (4) as shown in the equation at the bottom of the page, where we used the

$$
\begin{aligned}
\int_{-\ell_{x} / 2}^{\ell_{x} / 2} \int_{-\ell_{z} / 2}^{\ell_{z} / 2} \frac{d x_{2} d z_{2}}{\sqrt{\left(x_{1}-x_{2}\right)^{2}+y_{1}^{2}+\left(z_{1}-z_{2}\right)^{2}}} & =\int_{-\ell_{x} / 2}^{\ell_{x} / 2} \ln \frac{\left(\frac{\ell_{z}}{2}-z_{1}\right)+\sqrt{\left(x_{1}-x_{2}\right)^{2}+y_{1}^{2}+\left(\frac{\ell_{z}}{2}-z_{1}\right)^{2}}}{-\left(\frac{\ell_{z}}{2}+z_{1}\right)+\sqrt{\left(x_{1}-x_{2}\right)^{2}+y_{1}^{2}+\left(\frac{\ell_{z}}{2}+z_{1}\right)^{2}}} d x_{2} \\
& \approx \int_{-\ell_{x} / 2}^{\ell_{x} / 2} \ln \frac{\ell_{z}^{2}}{\left(x_{1}-x_{2}\right)^{2}+y_{1}^{2}} d x_{2}
\end{aligned}
$$


approximation given by (3). Now we calculate the last integral, yielding

$$
\begin{aligned}
& \int_{-\ell_{x} / 2}^{\ell_{x} / 2} \ln \frac{\ell_{z}^{2}}{\left(x_{1}-x_{2}\right)^{2}+y_{1}^{2}} d x_{2} \\
& =-2 \ell_{x}-2\left|y_{1}\right|\left(\arctan \frac{\ell_{x} / 2-x_{1}}{\left|y_{1}\right|}+\arctan \frac{\ell_{x}+x_{1}}{\left|y_{1}\right|}\right) \\
& \quad+\frac{\ell_{x}}{2}\left[\ln \frac{\ell_{z}^{2}}{\left(\ell_{x}-x_{1}\right)^{2}+y_{1}^{2}}+\ln \frac{\ell_{z}}{\left(\ell_{x}+x_{1}\right)^{2}+y_{1}^{2}}\right] \\
& \quad+x_{1} \ln \frac{\left(\ell_{x} / 2-x_{1}\right)^{2}+y_{1}^{2}}{\left(\ell_{x} / 2+x_{1}\right)^{2}+y_{1}^{2}} \approx 2 \ell_{x} \ln \frac{2 \ell_{z}}{\ell_{x}}
\end{aligned}
$$

where again we used the approximation (3). The integral can then be given by

$$
\begin{gathered}
\int_{-\ell_{x} / 2}^{\ell_{x} / 2} \int_{-\ell_{z} / 2}^{\ell_{z} / 2} \frac{d x_{2} d z_{2}}{\sqrt{\left(x_{1}-x_{2}\right)^{2}+y_{1}^{2}+\left(z_{1}-z_{2}\right)^{2}}} \\
\approx 2 \ell_{x} \ln \frac{2 \ell_{z}}{\ell_{x}} \approx 2 \ell_{x} \ln \frac{\ell_{z}}{\ell_{x}} .
\end{gathered}
$$

This is a good approximation for $\ell_{z}>1000 \ell_{x}$.

\section{REFERENCES}

[1] C. Jungnickel and R. McCormmach, Intellectual Mastery of Nature-Theoretical Physics from Ohm to Einstein. Chicago, IL: Univ. Chicago Press, 1986, vol. 1-2.

[2] L. Rosenfeld, "The velocity of light and the evolution of electrodynamics," Il Nuovo Cimento, vol. 4, pp. 1630-1669, 1957.

[3] —_, "Kirchhoff, Gustav Robert," Dictionary of Scientific Biography, vol. 7, pp. 379-383, 1973.

[4] E. T. Whittaker, A History of the Theories of Aether and Electricity, volume 1: The Classical Theories. New York: Humanities Press, 1973.

[5] A. K. T. Assis, Weber's Electrodynamics. Dordrecht, The Netherlands: Kluwer Academic Publishers, 1994.

[6] G. Kirchhoff, "Ueber die Bewegung der Elektricität in Drähten," Ann. der Phys., vol. 100, pp. 193-217, 1857.

[7] — "Ueber die Bewegung der Elektricität in Leitern," Annalen der Physik, vol. 102, pp. 529-544, 1857.

[8] G. Kirchhoff, "On the motion of electricity in wires," Philo. Mag., vol. 13, pp. 393-412, 1857.

[9] P. Graneau and A. K. T. Assis, "Kirchhoff on the motion of electricity in conductors," Apeiron, vol. 19, pp. 19-25, 1994.
[10] — - "Elektrodynamische Maassbestimmungen insbesondere über elektrische Schwingungen," Abh. sächs. Ges. Wiss., vol. 6, pp. 105-241, 1864.

[11] J. P. Wesley, "Weber electrodynamics, Part I. General theory, steady current effects," Found. Phys. Lett., vol. 3, pp. 443-469, 1990.

[12] —-, "Weber electrodynamics, Part II. Unipolar induction, Zantenna," Found. Phys. Lett., vol. 3, pp. 471-490, 1990.

[13] J. P. Wesley, "Weber electrodynamics, Part III. Mechanics, gravitation," Found. Phys. Lett., vol. 3, pp. 581-605, 1990.

[14] T. E. Phipps Jr., "Toward modernization of Weber's force law," Phys. Essays, vol. 3, pp. 414-420, 1990.

[15] - "Weber-type laws of action-at-a-distance in modern physics," Apeiron, vol. 8, pp. 8-14, 1990.

[16] J. P. Wesley, Selected Topics in Advanced Fundamental Physics. Blumberg, Germany: Benjamin Wesley, 1991.

[17] T. E. Phipps Jr., "Derivation of a modernized Weber force law," Phys. Essays, vol. 5, pp. 425-428, 1992.

[18] A. K. T. Assis, "Changing the inertial mass of a charged particle," $J$. Phys. Soc. Jpn., vol. 62, pp. 1418-1422, 1993.

[19] E. T. Kinzer and J. Fukai, "Weber's force and Maxwell's equations," Found. Phys. Lett., vol. 9, pp. 457-461, 1996.

[20] D. M. Drury, "The electromagnetic momentum of a charged particle in Weber's theory," Galil. Electrodyn., vol. 8, pp. 75-78, 1997.

[21] J. Fukai and E. T. Kinzer, "Compatibility of Weber's force with Maxwell's equations," Galil. Electrodyn., vol. 8, pp. 53-55, 1997.

[22] J. Guala-Valverde, "Gravitational mutual energy in Weber-type mechanics," Phys. Essays, vol. 11, pp. 164-165, 1998.

[23] A. K. T. Assis, W. A. Rodrigues Jr., and A. J. Mania, "The electric field outside a stationary resistive wire carrying a constant current," Found. Phys., vol. 29, pp. 729-753, 1999.

[24] A. K. T. Assis, Relational Mechanics. Montreal, QC, Canada: Apeiron, 1999.

[25] J. Guala-Valverde, Inercia y Gravitacion. Neuquen, Argentina: Fundacion Julio Palacios, 1999.

[26] V. F. Mikhailov, "The action of an electrostatic potential on the electron mass," Ann. Fond. Louis Broglie, vol. 24, pp. 161-169, 1999.

[27] A. K. T. Assis, "Weber's law and mass variation," Phys. Lett. A, vol. 136, pp. 277-280, 1989.

[28] - "Can a steady current generate an electric field?," Phys. Essays, vol. 4, pp. 109-114, 1991.

[29] - "Centrifugal electrical force," Commun. Theor. Phys., vol. 18, pp. 475-478, 1992.

[30] A. K. T. Assis and D. S. Thober, "Unipolar induction and Weber's electrodynamics," in Frontiers of Fundamental Physics, M. Barone and F. Selleri, Eds. New York: Plenum, 1994, pp. 409-414.

[31] A. K. T. Assis, "Circuit theory in Weber electrodynamics," Eur. J. Phys., vol. 18, pp. 241-246, 1997.

[32] A. Sommerfeld, Electrodynamics. New York: Academic, 1964.

[33] L. D. Landau, E. M. Lifshitz, and L. P. Pitaevskii, Electrodynamics of Continuous Media, 2nd ed. Oxford, U.K.: Pergamon, 1984. 